\title{
Accessibility manager: Creating a profile of a new profession
}

\author{
Xiaochun Zhang \\ University of Bristol, United Kingdom \\ xiaochun.zhang@bristol.ac.uk \\ https://orcid.org/0000-0001-6334-6525
}

\begin{abstract}
Disability is viewed as part of the human condition. Measures aimed at promoting accessibility ensure that individuals with a disability can participate fully in society and on an equal footing with those who do not have a disability. Accessibility has been prioritized through regulations both nationally and globally. The Accessible Culture and Training project was funded by the ERASMUS + Programme of the European Union; i it promoted equal opportunities and access to cultural events across countries and languages. One of the main objectives of the project was to design a new job role entitled Accessibility Manager and to provide training associated with the position. This article reports on some of the intellectual outputs from the project, explains the process of defining the new job profile and discusses the skill set that has been identified. It concludes that the Accessible Culture and Training project responds appropriately to the formation of the new discipline, Accessibility Studies.
\end{abstract}

Keywords: accessibility, training, accessibility manager, Accessibility Studies

\section{Introduction}

Disability is considered to be part of the human condition. Fifteen per cent of the world's population is estimated to live with disability in some form, according to the World Health Organization (WHO) (World Health Organization, 2018). People in old age may experience increasing difficulties with functioning in one way or another. The International Classification of Functioning, Disability and Health (ICF), the WHO framework for measuring health and disability at both the individual and the population level, recognizes functioning and disability as "a dynamic interaction between health conditions and contextual factors, both personal and environmental" (World Health Organization, 2011, p. 4). The ICF categorizes problems with human functioning into the following three interconnected areas:

- Impairments are problems in body function or alterations in body structure: for example, paralysis or blindness;

- Activity limitations are difficulties in executing activities: for example, walking or eating;

- Participation restrictions are problems with involvement in any area of life: for example, facing discrimination in employment or transportation. 
Zhang, X. (2019). Accessibility manager: Creating a profile of a new profession. Linguistica Antverpiensia, New Series: Themes in Translation Studies, 18, 73-86.

Disability is defined by the ICF as an umbrella term which refers to difficulties encountered in any or all three areas of functioning (World Health Organisation, 2011, p. 5).

Accessibility is a precondition intended to ensure that persons with a disability enjoy full and equal participation in society. Accessibility is the 'ability to access'. It primarily refers to designs for people with disabilities, but research and development on accessibility in recent years has focused on equal access for everyone to social, cultural, political and economic life (Disability Information Bureau, n.d.). This includes not only physical but also digital access to the same tools, services, organizations and facilities (United Nations, 2013). Access is an essential requirement for respecting the human dignity of all (Greco, 2016).

Accessibility has been prioritized through regulations introduced at both the national and the international level. The United Nations Convention on the Rights of Persons with Disabilities (CRPD) was the first document intended as an international human rights instrument that establishes legally binding minimum standards for the rights of people with disabilities. It was adopted on 13 December 2006 and entered into force on 3 May 2008. Most governments around the globe endorsed the CRPD. There were 82 signatories to the Convention, 44 signatories to the Optional Protocol and one ratification of the Convention on 30 March 2007 (the day on which it was open for signature). The Convention reaffirms that "all persons with all types of disabilities must enjoy all human rights and fundamental freedoms" (United Nations, n.d.). Individuals with disabilities are no longer perceived as being "'objects' of charity, medical treatment and social protection" but as "subjects" with rights, who are capable of claiming those rights and making decisions for their lives based on their free and informed consent as well as being active members of society (United Nations, 2006).

At the national level, several countries have introduced legislation on accessibility: for example, the Disabilities Act of 1990 in the United States, the Disability Discrimination Act 1992 in Australia and the Equality Act 2010 in the United Kingdom. In Europe, the European Accessibility Act (EAA) was approved in March 2019; it aims to "improve the functioning of the internal market for accessible products and services by removing barriers created by divergent legislation" (European Commission, n.d.). The Act uses a set of common accessibility requirements at the EU level for a number of products and services, including:

- computers and operating systems

- ATMs and ticketing and check-in machines

- smartphones

- TV equipment related to digital television services

- telephony services and related equipment

- audiovisual media services such as television broadcast and related consumer equipment

- services related to air, bus, rail and waterborne passenger transport

- banking services

- e-books

- e-commerce.

The EAA creates an obligation for the Member States to ensure that the selected products and services placed in the internal market fulfil the accessibility requirements. In addition, the Act 
enables disabled and elderly people in the EU to benefit from more accessible products and services that can be offered at more competitive prices.

In support of the national and international legislation on accessibility, several projects were funded by the European Commission to consider accessibility-related issues and services. The Audio Description: Lifelong Access for the Blind (ADLAB) project (http://www.adlab project.eu/) designed guidelines for the practice of film audio description (AD). The ADLAB PRO project (https://www.adlabpro.eu/), the natural successor to ADLAB, aimed to create free open-access and flexible didactic materials for the audio describer, a crucial yet littleknown professional figure in the field of media accessibility. The SUMAT project (http://www. fp7-sumat-project.eu/) investigated the possibility of using machine translation (MT) together with post-editing for interlingual subtitling. The SAVAS project (http://www.fp7-savas.eu) built domain-specific Large Vocabulary Continuous Speech Recognizers (LVCSR) to develop automated intralingual subtitling. These projects focused on particular areas of research, namely AD, interlingual subtitling and intralingual subtitling. However, a holistic approach was needed urgently to promote the existing methods in order to enhance accessibility and the training of specialists in the field.

Against this background, the Accessible Culture and Training (ACT) project was funded by the European Commission and lasted from September 2015 to August 2018. The project aimed to restore the concept of equal opportunity and the human right of access to culture. It comprised a multinational and multisectoral consortium with a balanced participation of four universities (Autonomous University of Barcelona, Spain; Antwerp University, Belgium; Queen's University Belfast, United Kingdom; and the University of Vienna, Austria), two theatres (Trànsit Project, Spain and NT Gent, Belgium), two departments of local government (Department of Culture of the Government of Catalonia and Inter, the Flemish Centre of Expertise on Accessibility and Universal Design), and one quality agency (European Certification and Qualification Association, ECQA). Several end-user associations and other theatres in Europe also participated in the project voluntarily.

The main objective of the project was to define the profile of the Accessibility Manager for the Scenic Arts, a new profession, and to provide various types of training activity associated with it. The project included eight intellectual outputs, namely (1) accessibility profiling; (2) manager profile definition; (3) learning curriculum proposal at university level; (4) development, testing and implementation of a MOOC; (5) certification; (6) co-production; (7) Development of guidelines for the implementation of policy strategies in the field of accessibility for the scenic arts, and (8) quality label proposal. This article reports primarily the second stage the ACT project (intellectual output 2), which developed the skill set for the new job profile. It discusses the process and methodology employed and explains the skill set identified. In addition, it shares the experience obtained from the ACT project in the hope of benefiting others in the future development of media accessibility training.

\section{The process of creating a skill set for the Accessibility Manager}

The development of the skill set was completed in the six months from July to December 2016. Its creation is based on the research findings of the study conducted in the first stage of the project (intellectual output 1), expert reflection from the multinational and multisectoral team, and the ECQA standard. 
Zhang, X. (2019). Accessibility manager: Creating a profile of a new profession. Linguistica Antverpiensia, New Series: Themes in Translation Studies, 18, 73-86.

\subsection{Accessibility profiling}

The study conducted into intellectual output 1 (IO1) explored the current landscape in the scenic arts in the partner countries across Europe regarding accessibility issues and relevant training. The research focus was set in the field of scenic arts because major broadcasters had devoted accessibility specialists to make their content, experiences and tools accessible to the broadest possible audience and substantial scholarly attention had been dedicated to the accessibility issues in the translation and distribution of film and TV products. However, in the field of scenic arts, this job role had not been established and more research was urgently needed to take on issues in this field. One of the objectives of 101 was to strengthen links between researchers and the various stakeholders in the process of providing access to the scenic arts to all. To achieve this, an ethnographically oriented research approach was adopted, using a mixed-methods design. The study combined questionnaires, interviews and focus groups to collect both quantitative and qualitative data. The research was designed to investigate accessibility issues from the viewpoints of (1) arts venues, (2) users and (3) the artistic team.

For the arts venues, a survey was designed and distributed among various cultural venues to investigate the accessibility facilities and training currently available in the context of live performances. The specific issues examined included these:

1. To what extent are the various access facilities offered in the different arts venues in each partner country, and how does this vary?

2. How are access facilities promoted?

3. Who is responsible for accessibility?

4. What access quality control measures are in place?

5. What type of accessibility training is offered, if any?

6. What challenges are faced by venues regarding accessibility?

For the end-users, two methods of data collection were used in order to gather both qualitative and quantitative data:

- Questionnaire: a survey was designed, asking the general public about their experiences of accessibility to the arts; and

- Focus groups: these were organized as a complementary method of data collection.

For the artistic team, interviews and focus-group discussions were conducted to investigate the experiences, awareness, approaches and attitudes regarding accessibility on the part of other key stakeholders in the production of a cultural performance (e.g., director, dramaturge, production manager, sound designer, lighting designer, overall artistic technical coordinator and actor). All the questions were explored with a view to informing the development of the Accessibility Manager profile and the training to be developed later in the project.

The findings highlighted an urgent need for more awareness of accessibility issues and relevant training, which justified the ACT project's main objective of establishing a training programme. The results of this study were mapped into the design of the skill set for the Accessibility Manager and the content of training for the new profession. The full report of 
Zhang, X. (2019). Accessibility manager: Creating a profile of a new profession. Linguistica Antverpiensia, New Series: Themes in Translation Studies, 18, 73-86.

the study is available on the website of the project (http://pagines.uab.cat/act/content/io1accessibility-profiling).

\subsection{Expert reflection}

Based on the research findings of IO1, the team of the ACT project started developing the skill set according to their expertise. Abstracting reflections by experts in the field on what should be necessary for a job role is a traditional method for defining skill sets. For example, the competence framework of the European Master's in Translation (EMT) was established by an expert group of eight academics. The practice of expert reflection can be viewed as a kind of practical empiricism where knowledge is generated through experience (Pym, 2013).

The multinational and multisectoral research group of the ACT project consisted of 20 core members. The researchers from the four university partners are experts engaged in accessibility research and experienced trainers in higher education; they contributed to the skill set from the educator's perspective. The two theatres had acquired hands-on experience on accessibility through their daily work. They provided insights into what was required to facilitate accessible performance and what an Accessibility Manager should do from the employers' perspectives. The two government departments provided guidance on accessibility from the viewpoint of policy-making. The quality agency ensured that the skill set was developed according to the European standard. The skill set was initially drafted by the university partners and then circulated among all the partners for suggestions and amendments. The final version was developed after six rounds of feedback.

\subsection{ECQA Standard}

The job profile of Accessibility Manager was defined according to the standard and guidelines of the ECQA (European Certification and Qualification Association). The ECQA requires the job role description to comply with the European standards for skills descriptions before it can be accepted by the European skills portals. The skill sets for the Accessibility Manager were organized in the following format, as shown in Figure 1.

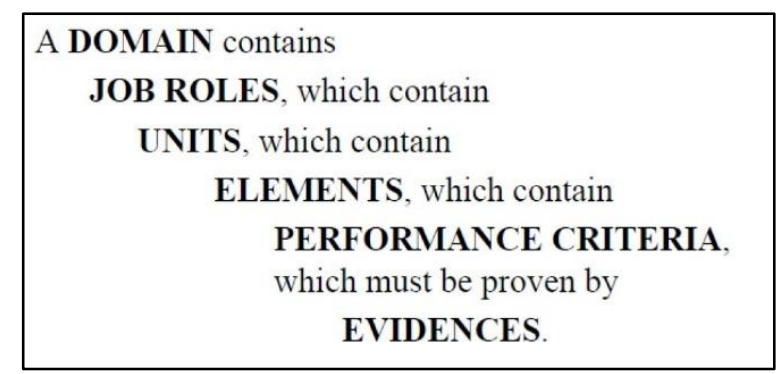

Figure 1 Skill Card/Skill Set Structure (Rules and Process Steps for Certification of Job Roles, ECQA 2011, p. 2).

In the ACT project, the domain was accessibility management and the consequent job role was the Accessibility Manager. The job role contained six learning units, which contain 26 elements reflected in 71 performance criteria. The evidence to demonstrate the fulfilment of the performance criteria was provided in the form of a massive open online course (MOOC) developed in a later stage of the project in intellectual output 4 (IO4). The MOOC entitled Accessibility to the Scenic Arts was created and is now available on Coursera, one of the major 
Zhang, X. (2019). Accessibility manager: Creating a profile of a new profession. Linguistica Antverpiensia, New Series: Themes in Translation Studies, 18, 73-86.

MOOC platforms (https://www.coursera.org/learn/accessibility-scenic-arts). The ECQA structure was applied in the formatting of the skill set for the Accessibility Manager (see Figure 2 as an example).

The final version of the skill set was validated by representatives of all the partners at the transnational project meeting in Vienna on 20 December 2016.

\section{Skills set of the Accessibility Manager}

In the process of profiling the skill set of the Accessibility Manager, two possible working contexts were identified. One was that the specialist works at a venue, such as a theatre. They take care of accessibility issues for events hosted there to ensure that the patrons have full access to various cultural performances. Meanwhile, there can be another scenario in which the specialist oversees broader cultural event management with a focus on accessibility. This person is not based in any given venue but operates at a regional level. After several rounds of discussion in the research group, the first role was defined as Accessibility Coordinator, while the second role was profiled as Accessibility Manager. The two job roles share a lot of similarities and most of the skills required overlap. The key dissimilarity between the two lies in the context in which the two specialists work. More specifically, an Accessibility Manager is in charge of coordinating accessibility services at various venues within his or her local district or region, which can involve collaborating with Accessibility Coordinators at different venues where such a role exists. If there is no Accessibility Coordinator at any given venue, the Accessibility Manager will then administer accessibility needs across these sites. Therefore, they should have sufficient knowledge of accessibility issues to enable them to make decisions on accessibility policies and evaluate the adequacy of facilities when choosing venues or productions.

Six units of skills were designed for both job roles, namely, understanding accessibility, venue accessibility, accessibility services I, accessibility services II, accessibility management for live events and promoting accessibility. Under the six units, 69 skills were identified for the Accessibility Coordinator, while 71 skills were identified for the Accessibility Manager. The Accessibility Manager is required to have additional skills, since they need to transfer knowledge about accessibility issues to meet the requirements of different environments. This involves working with local teams at various venues, which also requires an Accessibility Manager to have excellent interpersonal skills. Greater research skills are also required because the person is expected to investigate and evaluate situations in different contexts.

Considering the limitations of this article, the following section explains the skillsii defined for the Accessibility Manager, the more complex job role.

\subsection{Understanding accessibility}

First of all, it is essential for an Accessibility Manager to understand what accessibility is and what is required to make an event accessible. Therefore, these two elements were defined in the first unit (see Figure 2). As mentioned before, accessibility primarily focuses on designs for people with disabilities, whereas inclusion "allows for people with disabilities to take advantage of the benefits of the same health promotion and prevention activities experienced by people who do not have a disability" (USA Government, 2019). The principle underlying 
this idea is the concept of universal design, which refers to "the design of products and environments to be usable by all people, to the greatest extent possible, without the need for adaptation or specialised design" (Connell et al., 1997). An Accessibility Manager needs to understand these concepts fully and also the context of accessibility management.

In addition, in order to be able to manage an accessible event, an Accessibility Manager should have a good understanding of the different types and degrees of disability, so that they can select corresponding services to meet diverse requirements. Moreover, an Accessibility Manager should be familiar with local regulations on disability inclusion to make sure that the cultural events are organized in compliance with the relevant national and international legislation.

\section{Accessibility Manager}

\begin{tabular}{|c|c|c|c|}
\hline \multicolumn{4}{|c|}{ SKILL UNIT (U) } \\
\hline \multirow[t]{11}{*}{ ACT.U1 } & \multicolumn{3}{|c|}{ UNDERSTANDING ACCESSIBILITY } \\
\hline & \multicolumn{2}{|c|}{ Learning Element (E) } & \\
\hline & ACT.U1.E1 & \multicolumn{2}{|c|}{ What is Accessibility? } \\
\hline & & \multicolumn{2}{|c|}{ Performance Criteria (PC) } \\
\hline & & ACT.U1.E1.PC1 & S/he understands the basic concepts of inclusion and accessibility. \\
\hline & & ACT.U1.E1.PC2 & S/he understands the requirements for an accessible event. \\
\hline & ACT.U1.E2 & \multicolumn{2}{|c|}{ What is an Accessible Event? } \\
\hline & & ACT.U1.E2.PC1 & S/he understands the basic types and degrees of disability. \\
\hline & & ACT.U1.E2.PC2 & S/he understands the basic concepts of accessibility and its different forms. \\
\hline & & ACT.U1.E2.PC3 & S/he understands that there are national and international legislation and other requirements. \\
\hline & & ACT.U1.E2.PC4 & S/he demonstrates critical understanding of the concept of accessibility for everyone. \\
\hline
\end{tabular}

Figure 2 Skill set of Unit 1: Understanding Accessibility.

\subsection{Venue accessibility}

Unit two comprises a set of skills focusing on accessibility considerations for both indoor and outdoor venues (see Figure 3). Venue accessibility is the first step towards a successful inclusive cultural event. An Accessibility Manager should know the requirements for accessible facilities, such as parking spaces for disabled patrons, toilets, seating, shelters, lighting and furniture. For example, an accessible toilet is designed to accommodate wheelchair users, which requires more space than normal toilets. It should have a higher toilet pan, grab rails, shelves for colostomy and general use, a basin with lever or sensor taps and an emergency alarm for assistance. An Accessibility Manager needs to know the design standard of the accessible toilet to arrange such facilities for outdoor venues if there are none. In addition, service animals, such as guide dogs, need to be taken care of properly. An Accessibility Manager should make sure conveniences such as blankets, dog baskets and water bowls are prepared in case they are needed.

Moreover, the Accessibility Manager should understand the architectural risks of a venue and areas where improvements can be made. Some venues are historic buildings without modern facilities; the existing infrastructure and facilities cannot adequately meet the accessibility requirements. An Accessibility Manager should be able to identify the limits, help the venue to find temporary solutions and make long-term plans for future improvements. 
Zhang, X. (2019). Accessibility manager: Creating a profile of a new profession. Linguistica Antverpiensia, New Series: Themes in Translation Studies, 18, 73-86.

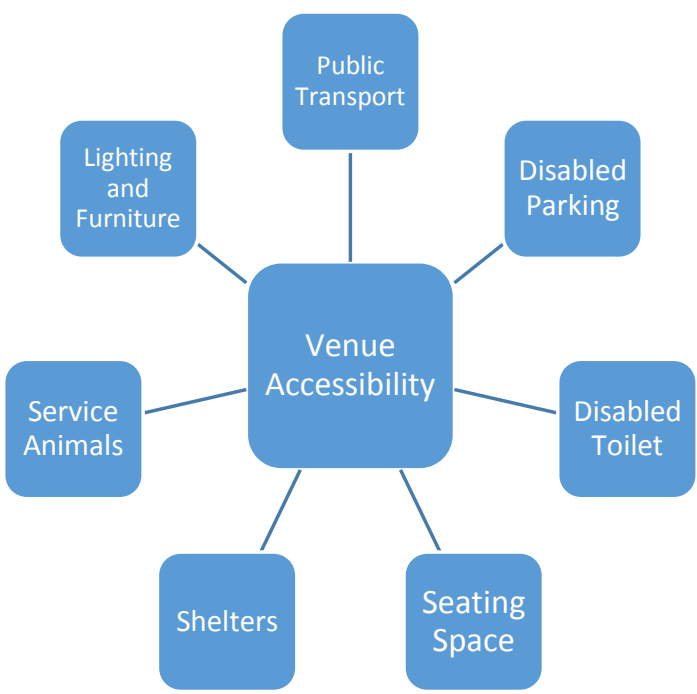

Figure 3 Elements to be considered for venue accessibility.

\subsection{Accessibility services}

This section discusses the skills related to accessibility services.ii An Accessibility Manager should have a good knowledge of the accessibility technology and services that are currently available, and their applicable scenarios. The existing accessibility services are presented in Figure 4.

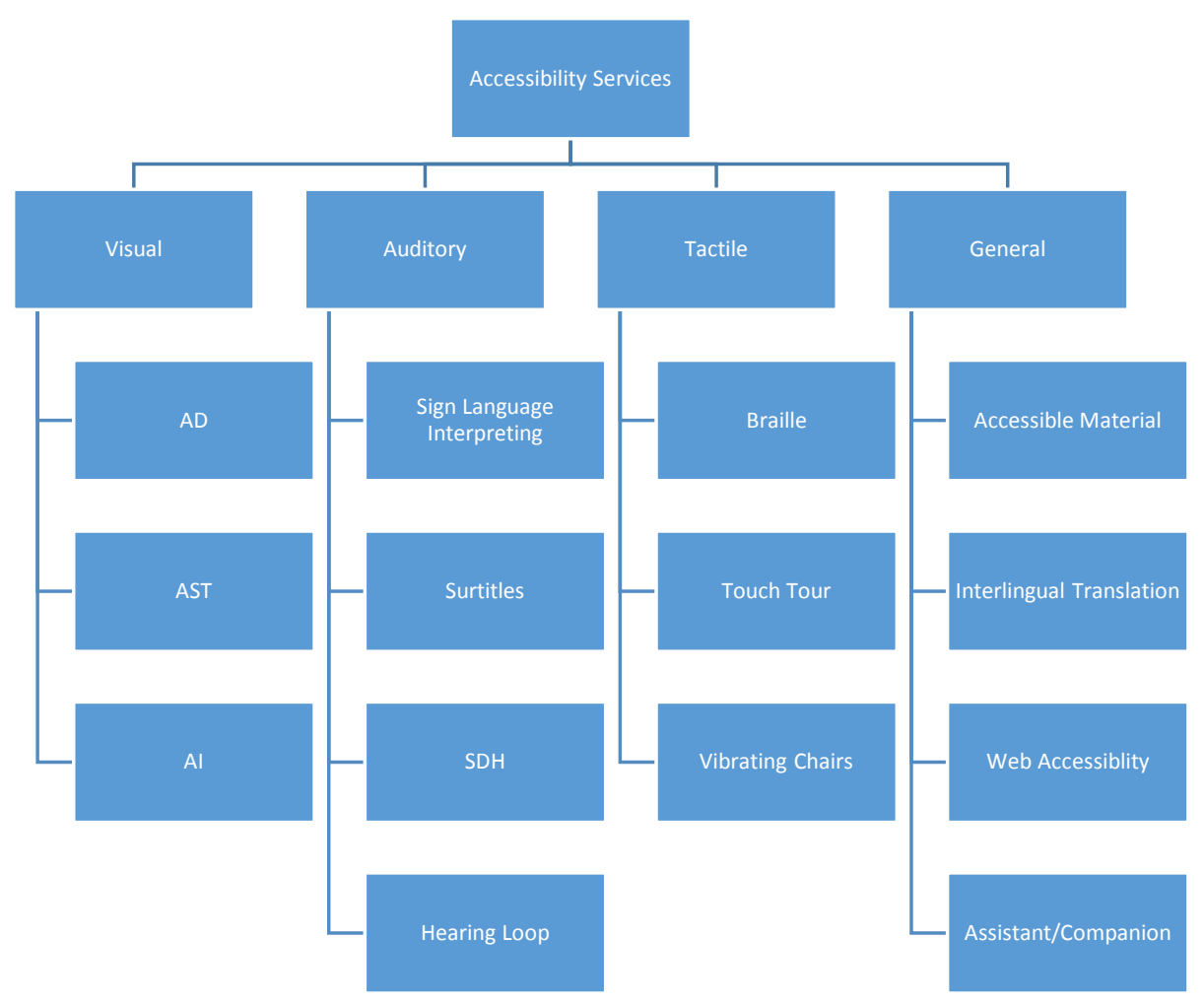

Figure 4 Accessibility services currently available.

These accessibility services and the technology can be categorized into four types of support, namely the visual, auditory, tactile and general. Support for access to visual information can 
Zhang, X. (2019). Accessibility manager: Creating a profile of a new profession. Linguistica Antverpiensia, New Series: Themes in Translation Studies, 18, 73-86.

help people who are blind and visually impaired. The available services include (Remael, Reviers, \& Vercauteren, 2014):

- Audio description (AD), which offers a verbal description of the relevant (visual) components of a work of art or media product, so that blind and visually impaired patrons can fully grasp its form and content.

- Audio subtitling (AST), which is the spoken rendering of the written (projected) subtitles or surtitles with a filmed or live performance.

- Audio introduction (AI), which refers to a narration read out live or recorded about the performance or the event that makes it easier for the audience to grasp the content of the production.

The services for access to auditory information can support people who are deaf or hard of hearing. The available services include (Matamala \& Orero, 2010):

- Sign language interpreting, which refers to the translation between a spoken and a signed language.

- Surtitles (or captions), which refers to the subtitles projected above the stage, which offer a brief translation of the dialogues into the language of the audience and are normally used for performances in a foreign language.

- Subtitles for the deaf and hard of hearing (SDH), which refers to subtitles that include not only the translation or transcription of the dialogue but also the descriptions of important sound effects, such as descriptions of music genres and information about who is speaking, when several speakers are on stage simultaneously.

- Hearing loop (or induction loop), which refers to a sound system that amplifies the sound coming from the stage.

Tactile services refer to support through the sense of touch. The available services include:

- Braille, which refers to the system of raised dots that people can read with their fingers.

- Touch Tour, which offers a tactile exploration of objects such as replicas, models and props, to help visually impaired people to complete their mental image of the things they touch.

- Vibrating chairs, which convert sound into vibrations that people can feel through their body and fingertips.

In addition to the three channels, there are services that provide additional support; these include:

- Accessible material, which refers to large print documents that use a point size of 16 and above, and Easy Read materials, which use pictures to support the meaning of the text.

- Interlingual translation, which refers to translation and/or interpretation between different languages.

- Web accessibility, which refers to the inclusive practice that ensures there are no barriers which prevent people with disabilities from interaction with or access 
Zhang, X. (2019). Accessibility manager: Creating a profile of a new profession. Linguistica Antverpiensia, New Series: Themes in Translation Studies, 18, 73-86.

to websites. The Web Content Accessibility Guidelines (WCAG) provide detailed information on this issue (see https://www.w3.org/TR/2018/REC-WCAG2120180605/).

- Assistant/companion, which refers to a human assistant or companion that a disabled person may have. It can be a professional carer, a family member or a friend.

These services can be combined to maximize the user experience. For example, when a blind audience goes to a theatre to see a play in a foreign language, a touch tour to the stage and the set can be arranged to provide them with an overview of the performance and enable them to explore the space. In addition, they can touch the props, costumes and furniture, which helps them to obtain more detail and to engage better with the performance. Furthermore, information in Braille and/or an audio introduction can be provided before the event starts in order to firm up the descriptive information that the blind person has received during the touch tour. During the performance, AD and audio subtitles can be provided to help them follow the dialogues in their native language and access visual elements such as the movements of the actors or changes of the set and/or costumes.

An Accessibility Manager needs to know how different accessibility services can be mixed and combined to provide the user with an optimized experience. They should have a good knowledge of the current accessibility services and devices available at the venue and know how to choose additional accessibility products and service providers based on the situation of each event and at each venue.

In addition, an Accessibility Manager needs to know the competences and skills of the providers of these services, so that they can make wise decisions when selecting the services for a particular event. For example, the professional who provides audio introductions, ADs, audio subtitling and auditory support for touch tours is an audio describer. A good audio describer should have the following competences (Matamala \& Orero, 2007, p. 330):

- the ability to undertake intersemiotic translations (turning images into words), both intralinguistically and interlinguistically, depending on the task commissioned;

- an excellent command of language and conventions;

- the ability to summarise information in order to adapt the text to the limited space available, keeping the original meaning, by means of rewording and by using synonyms;

- the ability to adapt the linguistic style to the target audience and to the product, by mastering different linguistic registers;

- the ability to critically select the most relevant information;

- in live $A D$, good oral expression and excellent diction.

An Accessibility Manager should be able to evaluate the competences of an audio describer, so as to decide whether or not to use his or her service. The same applies to all the other service providers. 
Zhang, X. (2019). Accessibility manager: Creating a profile of a new profession. Linguistica Antverpiensia, New Series: Themes in Translation Studies, 18, 73-86.

\subsection{Accessibility management for live events}

This section describes the management skills required for live events. The skill set is organized according to the process of arranging a live event, namely pre-event planning, coordination during the event, and post-event management.

Before the event, an Accessibility Manager should be able to coordinate the existing devices, technologies and software that provide accessibility solutions in the venue. They should make sure the facilities are in good order and ready to use. In addition, they should able to identify the accessibility needs of the target audiences and know how to plan and organize accessibility services based on potential demand. For example, an Accessibility Manager will be informed about the number of people with special needs coming to the event and the types of support they request. They should be able to evaluate whether the facilities at the venue are sufficient to meet all the needs of the audience. If the facilities do not, they should be able to order the services required and ensure that all the equipment is ready before the events. Importantly, an Accessibility Manager also needs to be able to calculate the costs of the accessibility services required and manage the financing with the venue and/or the live event organizer.

During the live event, an Accessibility Manager should support different accessibility service providers and handle common potential accidents and unexpected situations. For example, if a hearing loop stops working, the Accessibility Manager should know how to resolve the problem or find an alternative solution quickly.

After the event, an Accessibility Manager is expected to know how to organize the return of equipment and handle the payment of the service providers. Therefore, they should have some basic knowledge of accounting and finance. Moreover, they need to know how to analyse and summarize feedback from the end-users for future improvement. To improve the service, it is crucial that they take valuable advice from the customers, reflect on the strengths and weaknesses of current practice and compile action plans for future development. Based on the action plans, an Accessibility Manager should be able to organize and/or provide training for staff at various venues in preparation for hosting forthcoming events.

Over and above these skills, an Accessibility Manager should have some general managerial skills, which include:

- familiarity with the factors that promote good team communication and can implement them;

- knowing how to work with heterogeneous teams; here, they should be familiar with the factors that may lead to conflict and with measures to resolve conflict situations;

- the ability to assess the specific challenges of different venues, adapt to them and solve access issues;

- the ability to assess the know-how of local teams, collaborate with them and supply them with the required input on accessibility.

Since the Accessibility Manager does not work for an individual cultural venue but oversees the accessibility services in a region, they need to engage with different shareholders in the business and work with diverse teams at various venues. The venues may host artists and production companies from various countries in the world. An Accessibility Manager should 
Zhang, X. (2019). Accessibility manager: Creating a profile of a new profession. Linguistica Antverpiensia, New Series: Themes in Translation Studies, 18, 73-86.

therefore be able to manage a team with diverse sociocultural and linguistic backgrounds. Accordingly, they need to be flexible and ready to adapt to new teams, new conditions and new scenarios each time an event occurs.

\subsection{Promoting accessibility}

Other than managing accessible events, promoting accessibility is an essential part of the job role. First, an Accessibility Manager should know how to present the needs and benefits of organizing accessible events and argue the importance of accessibility. Although accessibility has been regulated nationally and internationally, awareness of accessibility issues and the availability of accessibility services need improving. The research findings from IO1 show that many venues have not made their events fully accessible or even accessible at all. Some offer accessible performances, but not regularly or not frequently enough to meet the users' demands. What is more, many decision-makers are not fully aware of the accessibility services available and how they can be used and combined to provide a better user experience. An Accessibility Manager should be able to help develop accessibility policy for venues in the region to enhance the development of an inclusive society and oversee the implementation of local regulations. To achieve this, they should know how to identify the relevant stakeholders and involve them. This could entail getting the relevant departments at a cultural venue to collaborate both internally and externally. An Accessibility Manager should forge connected or strengthening cooperation between existing organizations dedicated to disability issues and working closely with relevant local authorities. Meanwhile, making a cultural event accessible incurs costs for the venue and/or the production company. The Accessibility Manager should be aware of the financial implications of accessibility services and be able to present different solutions to the stakeholders based on their budgets.

Moreover, an Accessibility Manager should know how to promote an accessible event. The end-users do not always receive information about accessible events, partly because these events are not promoted through the channels of communication they use. An Accessibility Manager should know how to promote events through traditional media, such as newspapers, leaflets and posters, as well as via new channels, such as online and social media. However, they must make sure that all information is provided in an accessible way. For example, leaflets should be available in Braille or large print and web content should be created following the Web Content Accessibility Guidelines.

The skill set presented above was mapped to courses, learning outcomes and learning methods for the design of training programmes in the later stages of the project in $\mathrm{IO}$ and 104. Two curricula for post-graduate study at universities were created, facilitated by the approach of ECQA and by the Bologna process in IO3.iv One was the Master's Programme in Accessibility Management for the Scenic Arts, which comprised the profiles of both the Accessibility Manager and the Accessibility Coordinator. The MA programme was designed for a duration of 24 months with 120 European Credit Transfer and Accumulation System (ECTS) points. The other programme was a shorter post-graduate programme of 12 months totalling 60 ECTS points, which was designed for the profile of the Accessibility Coordinator. The two curriculum designs and suggested teaching materials were provided as a reference for any universities that are interested in starting new programmes on Accessibility Management to adopt for and adapt to their own needs. In addition to the training in the higher-education institutions, the aforementioned MOOC for Accessibility to the Scenic Arts was designed and 
Zhang, X. (2019). Accessibility manager: Creating a profile of a new profession. Linguistica Antverpiensia, New Series: Themes in Translation Studies, 18, 73-86.

launched in $\mathrm{IO} 4$ to provide training for lifelong learners. Regarding future employment, the graduate students can be hired by a cultural venue as Accessibility Coordinator, work as Accessibility Manager on a freelance basis or join local governments or non-governmental organizations (NGOs) that engage in disability care and services.

\section{Conclusion}

The Accessibility Manager is a new and highly responsible profession. The skills defined for the role are intended to serve as a starting point which is to be tested and improved in practice in the years to come, not only in Europe but also internationally. Access to culture for everyone is vital in the establishment of a more inclusive and diverse society that enriches humankind. The ACT project was completed in August 2018. However, the mission of improving accessibility does not end there.

Recent research in Translation Studies and the latest developments in translation practice have "not only led to a profusion of approaches, but also to the development of new text forms and translation modes" (Remael, Reviers \& Vandekerckhove, 2016, p. 248). Media Accessibility has attracted scholars from various fields, thanks to many international and interdisciplinary research projects, such as the ACT project. During the process of sharing research methods from divergent disciplines in these projects, scholars have gradually been moving beyond the boundaries of their traditional fields, such as Translation Studies, and forming a new community of Accessibility Studies, a research field engaged in "the critical investigation of accessibility processes and phenomena, and the design, implementation and evaluation of accessibility-based and accessibility-oriented methodologies" (Greco, 2018, p. 219). The ACT project is a timely response to the novel features of the new field and a point of departure for further development.

\section{References}

Connell, B. R., Jones, M., Mace, R., Mueller, J., Mullick, A., Ostroff, E., . . Vanderheiden, G. (1997). The principles of universal design [Report]. Retrieved from https://projects.ncsu.edu/ncsu/design/ cud/about_ud/udprinciplestext.htm

Disability Information Bureau. (n.d.). Accessibility issues. Retrieved from https://www.dibservices.org. uk/24-accessibility-issues

European Commission. (n.d.). European accessibility act. Retrieved from https://ec.europa.eu/social/ main.jsp?catld $=1202$

European Qualifications Framework. (2008). The European qualifications framework for lifelong learning [Leaflet]. Retrieved from https://ec.europa.eu/ploteus/sites/eac-eqf/files/leaflet_en. pdf

Greco, G. M. (2016). On accessibility as a human right, with an application to media accessibility. In A. Matamala \& P. Orero (Eds.), Researching audio description: New approaches (pp. 11-33). London, England: Palgrave Macmillan. doi:10.1057/978-1-137-56917-2_2

Greco, G. M. (2018). The nature of accessibility studies. Journal of Audiovisual Translation, 1(1), 205232.

Matamala, A., \& Orero, P. (2007). Designing a course on audio description and defining the main competences of the future professional. Linguistica Antverpiensia, New Series - Themes in Translation Studies, 6, 329-344.

Matamala, A., \& Orero, P. (Eds.). (2010). Listening to subtitles: Subtitles for the deaf and hard of hearing. Bern, Switzerland: Peter Lang. doi:10.3726/978-3-0351-0147-8 
Zhang, X. (2019). Accessibility manager: Creating a profile of a new profession. Linguistica

Antverpiensia, New Series: Themes in Translation Studies, 18, 73-86.

Pym, A. (2013). Translation skill-sets in a machine-translation age. Meta, 58(3), 487-503. doi:10.7202/ 1025047ar

Remael, A., Reviers, N., \& Vandekerckhove, R. (2016). From translation studies and audiovisual translation to media accessibility: Some research trends. Target. International Journal of Translation Studies, 28(2), 248-260. doi:10.1075/target.28.2.06rem

Remael, A., Reviers, N., \& Vercauteren, G. (2014). Pictures painted in words: ADLAB audio description guidelines [Report]. Retrieved from http://www.adlabproject.eu/Docs/adlab\%20book/index. html

United Nations. (n.d.). Convention on the rights of persons with disabilities (CRPD). Retrieved from https://www.un.org/development/desa/disabilities/convention-on-the-rights-of-persons-withdisabilities.html

United Nations. (2006). Convention on the rights of persons with disabilities and optional protocol [Report]. Retrieved from http://www.un.org/disabilities/documents/convention/convoptprote.pdf

United Nations. (2013). Accessibility and development: Mainstreaming disability in the post-2015 development agenda [Report]. Retrieved from http://www.un.org/disabilities/documents/ accessibility_and_development.pdf

USA Government. (2019). Disability inclusion. Retrieved from https://www.cdc.gov/ncbddd/disability andhealth/disability-inclusion.html

World Health Organisation. (2011). World report on disability [Report]. Retrieved from http://www. who.int/disabilities/world_report/2011/report.pdf

World Health Organisation. (2018). Disability and health. Retrieved from https://www.who.int/news$\mathrm{room} /$ fact-sheets/detail/disability-and-health

\footnotetext{
' The Accessible Culture and Training project was funded by the ERASMUS + Programme of the European Union under the call Knowledge Alliance 2 with the reference 2015-1-ES01-KA203-015734.

i" In the context of the ACT project, "skills" was defined according to the European Qualifications Framework (EQF). This refers to a skill as "the ability to apply knowledge and use know-how to complete tasks and solve problems" (EQF, 2008, p. 11). In EQK, skills are further described as "cognitive, involving the use of logical, intuitive and creative thinking or practical, involving manual dexterity and the use of methods, materials, tools and instruments" (EQF, 2008, p. 11).

iii In the ACT project, the section was divided into two units, namely units 3 and 4 , because of the requirement of the ECQA standard.

iv The full report on 103 and the curriculum design for the two programmes are available on the project's website at http://pagines.uab.cat/act/content/io3-learning-curriculum-proposal-universitylevel.
} 